\title{
Using strong inference to answer causal questions in spinal cord injury research
}

\author{
Jonviea D. Chamberlain ${ }^{1,2}$ - Martin W. G. Brinkhof $\mathbb{B}^{1,2}$
}

Received: 7 August 2019 / Accepted: 7 August 2019 / Published online: 22 August 2019

(c) International Spinal Cord Society 2019

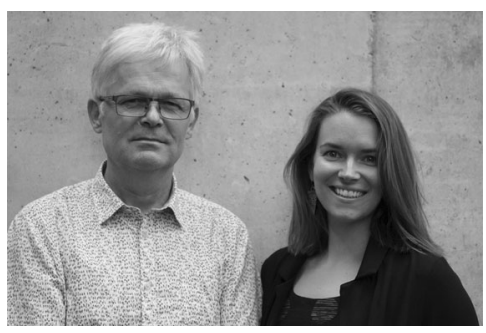

Following the recent call to arms against significance testing [1], supported by Spinal Cord [2], we have finally begun to shed our indoctrinated notions that only a significant $p$-value can confirm interesting and reportable research with notable findings. But, the revolution cannot stop there. Doing so would only lead to a replacement of the $p$-value with another lackadaisical methodology to make research "easy", and allow the perpetuation of lazy conclusionse.g., "...further research is needed." In the recent editorial of the American Statistician, authors call for a shift away from statistical significance towards "statistical thinking" [3]. To continue our progression towards superior, clinically relevant science, we here would like to draw attention to another key methodological overhaul-the shift from statistical inference towards causal inference. It is imperative that we move from descriptions to causal inference; from suggesting associations to inferring causality.

Causal inference is often the central objective in SCI research, and yet one that we are all afraid to articulate when using non-experimental study designs [4-6]. But, avoiding the so called "C-word" in discussions of observational research has restrained our thinking in terms of study design and objectives, allowing us to remain in a mire of "random medical news" related to improper study identification (i.e., of the hypotheses, objective, etc.), further propagated by the misuse of $p$-values. Every day we make implicit inferences about the world around us, but to move forward towards causal inference in our scientific research

Jonviea D. Chamberlain

jonviea.chamberlain@paraplegie.ch

$\bowtie$ Martin W. G. Brinkhof

martin.brinkhof@paraplegie.ch

Swiss Paraplegic Research, Nottwil, Switzerland

2 Health Sciences and Health Policy, University of Lucerne, Luzern, Switzerland we need to formalize our inferences. At first blush, three main hurdles stand in the way of observational research as a tool for causal inference: unmeasured confounding, collider bias, and sampling bias. Fortunately, new and old methodologies are available that can help address these challenges.

Directed acyclic graphs [DAGs] are tools commonly employed and now readily accessible for causal thinking exercises that can aid in formalizing causal inferences [7, 8]. DAGs are hypothesis-driven, graphical representations of the alleged relationship between the exposure and outcome of interest [7], and force researchers to clearly present all of their assumptions regarding this relationship. Furthermore, with unidirectional arrows, DAGs permit the visual representation of the supposed causal pathway so as to identify all potential confounders (measured and unmeasured) and colliders (for which inadvertent inclusion in study design and modelling would induce bias).

After the collection and analyses of data, many methodologies are available to assess the reliability of our results and conclusions in light of, e.g., sparse data vulnerable to sampling bias [9], unmeasured and unknown confounders [10], or potential selection bias. A major recent development includes estimating an $E$-value to assess the strength of evidence in light of potential unknown or unmeasured confounding [10]. To this effect, an $E$-value provides an estimate for the minimum strength of association needed for an unmeasured confounder to explain away an observed exposure-outcome association, conditional on the measured covariates [10]. In addition, it is possible to identify and partially quantify potential sampling bias-i.e., bias resulting from including a subset that is systematically different from the larger target population (e.g., selection bias)—and its impact on conclusions through sensitivity analyses. The full complexity and usage of sensitivity analyses goes beyond the scope of the present editorial, but such analyses can aid in evaluating the strength of results under alternative scenarios. 
Undertaking causal inference requires theory-based study design and a clearly defined causal question. However, regardless of how standardized and convincing our causal inferences may be, going hand-in-hand with causal inference should be "strong inference". Strong inference was proposed by Platt in a 1964 essay to be the reason behind certain scientific fields experiencing rapid progression while others stagnate [11]. In essence, strong inference is about providing evidence to rule out alternative explanations for the perceived or measured reality around us. Following the principles of strong inference, researchers must generate testable study hypotheses, including viable alternative hypotheses, for which welldesigned studies will exclude either the null hypothesis or the alternative hypothesis. The conclusion of the study would therefore clearly be able to falsify one of the proposed hypotheses. Encompassed within the construct of strong inference is the uncertainty of science, and thereby the falsification requirement for causal questions. Importantly, the null hypothesis is not referring to the statistical null hypothesis, but rather the hypothesis that follows from previous evidence and theory of why exposure $x$ would cause outcome $y$.

The causal revolution is mounting, with epidemiologists and other health scientists breaking free from dogmatic constraints (e.g., "correlation does not equal causation") and the restrictive thinking that only experimental research (e.g., randomized control trials) can help illuminate/ respond to questions of causality. To strengthen research in SCI, as health scientists we must stay open and willing to consistently challenge our previous findings, theories, and hypotheses; to incorporate the causal inference framework in our research practice; and to employ causal thinking. In an era sans $p$-values, we must follow the recommendations put forth by Wasserstein et al. to "[a]ccept uncertainty...[b]e thoughtful, open, and modest" [3] — and thereby practice strong inference when considering questions of causality.

Publisher's note: Springer Nature remains neutral with regard to jurisdictional claims in published maps and institutional affiliations.

\section{References}

1. Amrhein V, Greenland S, McShane B. Scientists rise up against statistical significance. Nature. 2019;567:305-7. https://doi.org/ 10.1038/d41586-019-00857-9.

2. Harvey LA, Brinkhof MWG. Imagine a research world without the words "statistically significant". Is it really Possible? Spinal Cord. 2019;57:437-8. https://doi.org/10.1038/s41393019-0292-2.

3. Wasserstein RL, Schirm AL, Lazar NA. Moving to a world beyond "p<0.05". Am Stat. 2019;73(Suppl 1):1-19. https://doi. org/10.1080/00031305.2019.1583913.

4. Galea S, Vaughan RD. Moving beyond the cause constraint: a public health of consequence, may 2018. Am J Public Health. 2018;108:602-3. https://doi.org/10.2105/ajph.2018.304390.

5. Hernan M. The c-word: the more we discuss it, the less dirty it sounds. Am J Public Health. 2018;108:625-6. https://doi.org/10. 2105/ajph.2018.304392.

6. Hernan MA. The c-word: scientific euphemisms do not improve causal inference from observational data. Am J Public Health. 2018;108:616-9. https://doi.org/10.2105/ajph.2018.304337.

7. Greenland S, Pearl J, Robins JM. Causal diagrams for epidemiologic research. Epidemiology 1999;10:37-48.

8. Textor J, Hardt J, Knuppel S. Dagitty: a graphical tool for analyzing causal diagrams. Epidemiology. 2011;22:745. https://doi. org/10.1097/EDE.0b013e318225c2be.

9. Discacciati A, Orsini N, Greenland S. Approximate bayesian logistic regression via penalized likelihood by data augmentation. Stata J. 2015;15:712-36.

10. VanderWeele TJ, Ding P. Sensitivity analysis in observational research: Introducing the E-value. Ann Intern Med. 2017; 167:268-74. https://doi.org/10.7326/m16-2607.

11. Platt JR. Strong inference: certain systematic methods of scientific thinking may produce much more rapid progress than others. Science. 1964;146:347-53. https://doi.org/10.1126/science.146. 3642.347. 